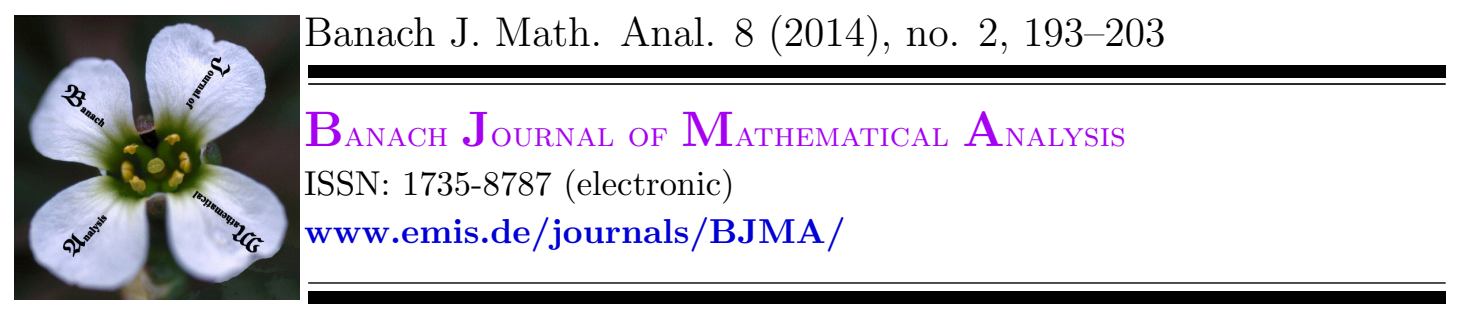

\title{
CERTAIN DISTANCE ESTIMATES FOR OPERATORS ON THE BERGMAN SPACE
}

\author{
NAMITA DAS ${ }^{1}$ AND MADHUSMITA SAHOO ${ }^{2 *}$ \\ Communicated by F. Kittaneh
}

\begin{abstract}
Let $\mathbb{D}$ be the open unit disk with its boundary $\partial \mathbb{D}$ in the complex plane $\mathbb{C}$ and $d A(z)=\frac{1}{\pi} d x d y$, the normalized area measure on $\mathbb{D}$. Let $L_{a}^{2}(\mathbb{D}, d A)$ be the Bergman space consisting of analytic functions on $\mathbb{D}$ that are also in $L^{2}(\mathbb{D}, d A)$. In this paper we obtain certain distance estimates for bounded linear operators defined on the Bergman space.
\end{abstract}

\section{INTRODUCTION}

Let $d A$ denote Lebesgue area measure on the unit disk $\mathbb{D}$, normalized so that the measure of $\mathbb{D}$ equals 1 . The Bergman space $L_{a}^{2}(\mathbb{D})$ is the Hilbert space consisting of the analytic functions on $\mathbb{D}$ that are also in $L^{2}(\mathbb{D}, d A)$. Since point evaluation at $z \in \mathbb{D}$ is a bounded linear functional on the Hilbert space $L_{a}^{2}(\mathbb{D})$, the Riesz representation theorem implies that there exists a unique function $K_{z}$ in $L_{a}^{2}(\mathbb{D})$ such that

$$
f(z)=\int_{\mathbb{D}} f(w) \overline{K_{z}(w)} d A(w)
$$

for all $f \in L_{a}^{2}(\mathbb{D})$. Let $K(z, w)$ be the function on $\mathbb{D} \times \mathbb{D}$ defined by $K(z, w)=$ $\overline{K_{z}(w)}=\frac{1}{(1-z \bar{w})^{2}}$. The function $K(z, w)$ is called the Bergman kernel of $\mathbb{D}$ or the

Date: Received: Sep. 13, 2013; Revised: Dec. 8, 2013; Accepted: Dec. 23, 2013.

* Corresponding author.

2010 Mathematics Subject Classification. Primary 47B15; Secondary 47B35.

Key words and phrases. Bergman space, positive operators, Toeplitz operators, bounded harmonic functions, distance estimates. 
reproducing kernel of $L_{a}^{2}(\mathbb{D})$ because the formula

$$
f(z)=\int_{\mathbb{D}} f(w) K(z, w) d A(w)
$$

reproduces each $f \in L_{a}^{2}(\mathbb{D})$. The sequence of functions $e_{n}(z)=\sqrt{n+1} z^{n}, n=$ $0,1,2, \ldots$ form an orthonormal basis for $L_{a}^{2}(\mathbb{D})$ and $K(z, w)=\sum_{n=0}^{\infty} e_{n}(z) \overline{e_{n}(w)}$. For $a \in \mathbb{D}$, let $\phi_{a}$ be the analytic map from $\mathbb{D}$ onto $\mathbb{D}$ defined by

$$
\phi_{a}(z)=\frac{a-z}{1-\bar{a} z}, z \in \mathbb{D} .
$$

Let $\mathcal{L}\left(L_{a}^{2}(\mathbb{D})\right)$ be the set of all bounded linear operators from $L_{a}^{2}(\mathbb{D})$ into itself. Let $\mathcal{L C}\left(L_{a}^{2}(\mathbb{D})\right)$ denote the ideal of compact operators in $\mathcal{L}\left(L_{a}^{2}(\mathbb{D})\right)$. Let $L^{\infty}(\mathbb{D})$ denote the usual space of bounded measurable functions $f$ on the unit disk $\mathbb{D}$ in the complex plane with $\|f\|_{\infty}=\operatorname{ess} \sup \{|f(z)|: z \in \mathbb{D}\}<\infty$. Let $H^{\infty}(\mathbb{D})$ be the space of bounded analytic functions on $\mathbb{D}$ and $h^{\infty}(\mathbb{D})$ be the space of all bounded harmonic functions on $\mathbb{D}$.

For $T \in \mathcal{L}\left(L_{a}^{2}(\mathbb{D})\right)$, the Berezin transform of $T$ is the function $\widetilde{T}$ on $\mathbb{D}$ defined by

$$
\widetilde{T}(z)=\left\langle T k_{z}, k_{z}\right\rangle
$$

where $k_{z}(w)=\frac{K(w, z)}{\sqrt{K(z, z)}}=\frac{1-|z|^{2}}{(1-\bar{z} w)^{2}}$. These functions are called normalized reproducing kernels of $L_{a}^{2}(\mathbb{D})$; it is clear that they are unit vectors in $L_{a}^{2}(\mathbb{D})$. For $\phi \in L^{\infty}(\mathbb{D})$, the Toeplitz operator with symbol $\phi$ is the operator $T_{\phi}$ on $L_{a}^{2}(\mathbb{D})$ defined by $T_{\phi} f=P(\phi f)$, where $P$ is the orthogonal projection of $L^{2}(\mathbb{D}, d A)$ onto $L_{a}^{2}(\mathbb{D})$. The Berezin transform of the function $\phi$, denoted by $\widetilde{\phi}$, is defined to be the Berezin transform of the Toeplitz operator $T_{\phi}$. This definition easily leads to the formula

$$
\widetilde{\phi}(z)=\left(1-|z|^{2}\right)^{2} \int_{\mathbb{D}} \frac{\phi(w)}{|1-\bar{z} w|^{4}} d A(w) .
$$

If $\phi \in h^{\infty}(\mathbb{D})$, then $\widetilde{T_{\phi}}=\widetilde{\phi}=\phi$. Let $\phi: \mathbb{D} \longrightarrow \mathbb{D}$ be analytic. Then $\phi$ induces a composition operator $C_{\phi}$ on $L_{a}^{2}(\mathbb{D})$ defined by $C_{\phi} f=f \circ \phi$, for all $f \in L_{a}^{2}(\mathbb{D})$. An operator $A \in \mathcal{L}\left(L_{a}^{2}(\mathbb{D})\right)$ is positive if $\langle A f, f\rangle \geq 0$, for every $f \in L_{a}^{2}(\mathbb{D})$. In this case we write $A \geq 0$. Let $\sigma(A)$ denote the spectrum of $A \in \mathcal{L}\left(L_{a}^{2}(\mathbb{D})\right)$. In section 2, we introduce the operator $U_{a} \in \mathcal{L}\left(L_{a}^{2}(\mathbb{D})\right), a \in \mathbb{D}$ and discuss certain algebraic properties of these classes of operators. In section 3 , we propose some distance estimates for operators defined on the Bergman space. In section 4, we are concerned only with Toeplitz operators with bounded harmonic symbols and obtained certain distance estimates between Toeplitz operators.

\section{Preliminaries}

In this section we introduce the operator $U_{a} \in \mathcal{L}\left(L_{a}^{2}(\mathbb{D})\right), a \in \mathbb{D}$ and discuss certain algebraic properties of these classes of operators. Given $a \in \mathbb{D}$, we define the operator $U_{a}$ from $L_{a}^{2}(\mathbb{D})$ into itself as $U_{a} f=\left(f \circ \phi_{a}\right) k_{a}$. The Proposition 2.1 
is well known. For example see [11]. To make the presentation self-contained, we give a proof of the proposition.

Proposition 2.1. Let $a \in \mathbb{D}$. The following hold:

(i) $U_{a}$ is self-adjoint unitary operator.

(ii) $U_{a} K_{z}=\overline{k_{a}(z)} K_{\phi_{a}(z)}$.

(iii) $U_{a} k_{z}=\alpha k_{\phi_{a}(z)}$ where $\alpha=\alpha_{a}(z) \in \partial \mathbb{D}$.

Proof. (i). Let $f, g \in L_{a}^{2}(\mathbb{D})$ and let $\phi_{a}$ be the Möbius transformation for $a \in \mathbb{D}$. Then

$$
\left\langle f \circ \phi_{a}, g \circ \phi_{a}\right\rangle=\left\langle k_{a} f, k_{a} g\right\rangle .
$$

First we will prove $U_{a}$ is self-adjoint. If $f, g \in L_{a}^{2}(\mathbb{D})$ then

$\left\langle U_{a}^{*} f, g\right\rangle=\left\langle f, U_{a} g\right\rangle=\left\langle f, k_{a}\left(g \circ \phi_{a}\right)\right\rangle=\left\langle k_{a} f \circ \phi_{a}, k_{a}\left(k_{a} \circ \phi_{a}\right) g\right\rangle=\left\langle U_{a} f,\left(k_{a}\left(k_{a} \circ \phi_{a}\right) g\right)\right\rangle$

where we have used the above change of variable and the identity $\phi_{a} \circ \phi_{a}(w)=w$. Since $\left(k_{a}\left(k_{a} \circ \phi_{a}\right)\right)=1$, so $\left\langle U_{a}^{*} f, g\right\rangle=\left\langle U_{a} f, g\right\rangle$. That $U_{a}$ is unitary follows similarly. Simply rewrite the change of variable replacing $f$ by $f \circ \phi_{a}$, simplify the result with the identity $k_{z} \circ \phi_{z}(w)=k_{z}(w)^{-1}$. Combining these result yields $U_{a}^{-1}=U_{a}^{*}=U_{a}$. This proves (i). For (ii), let $f \in L_{a}^{2}(\mathbb{D})$. Now

$$
\begin{aligned}
\left\langle f, U_{a} K_{z}\right\rangle & =\left\langle U_{a} f, K_{z}\right\rangle=U_{a} f(z)=k_{a}(z) f\left(\phi_{a}(z)\right) \\
& =k_{a}(z)\left\langle f, K_{\phi_{a}(z)}\right\rangle=\left\langle f, \overline{k_{a}(z)} K_{\phi_{a}(z)}\right\rangle .
\end{aligned}
$$

This proves (ii). We shall now prove (iii). Notice that

$$
U_{a} k_{z}=\frac{1}{\left\|K_{z}\right\|} U_{a} K_{z}=\frac{1}{\left\|K_{z}\right\|} \overline{k_{a}(z)} K_{\phi_{a}(z)}=\left(\frac{\left\|K_{\phi_{a}(z)}\right\|}{\left\|K_{z}\right\|} \overline{k_{a}(z)}\right) k_{\phi_{a}(z)} .
$$

Setting $\alpha=\alpha_{a}(z)=\left\|K_{\phi_{a}(z)}\right\|\left\|K_{z}\right\|^{-1} \overline{k_{a}(z)}$ we get the desired identity. Since

$$
1-\left|\phi_{a}(z)\right|^{2}=\frac{\left(1-|a|^{2}\right)\left(1-|z|^{2}\right)}{|1-\bar{a} z|^{2}}
$$

To prove $\alpha_{a}(z) \in \partial \mathbb{D}$ we calculate

$$
\alpha_{a}(z)=\frac{1-|z|^{2}}{1-\left|\phi_{a}(z)\right|^{2}} \frac{1-|a|^{2}}{(1-a \bar{z})^{2}}=\frac{|1-a \bar{z}|^{2}}{(1-a \bar{z})^{2}} .
$$

Now since $k_{a}(z) k_{a}\left(\phi_{a}(z)\right)=1$ for each $a \in \mathbb{D}$, hence $U_{a} k_{a}=1$ and $U_{a} k_{m_{a}}=$ $k_{m_{a}}$ where $m_{a}$ is the geodesic midpoint between 0 and $a$ (see [6]), i.e, $m_{a}=$ $\frac{1-\sqrt{1-|a|^{2}}}{|a|^{2}} a$. Further $U_{a} k_{z}=k_{\phi_{a}(z)}$.

Lemma 2.2. Let $T \in \mathcal{L}\left(L_{a}^{2}(\mathbb{D})\right)$. The following hold:

(i) If $T U_{a}=U_{a} T$ for all $a \in \mathbb{D}$, then $T=\alpha I$ for some $\alpha \in \mathbb{C}$.

(ii) If $T U_{\phi_{a}}=U_{\phi_{a}} T$ for all $a \in \mathbb{D}$, then $T=\beta I$ for some $\beta \in \mathbb{C}$.

(iii) If $T U_{a}=U_{a} T$ for some $a \in \mathbb{D}$, then $M_{a}=\left\{k_{m_{a}}\left(g \circ \phi_{m_{a}}\right): g\right.$ even $\}$ is a reducing subspace of $T$.

Proof. For proof see [8]. 
Lemma 2.3. Let $T \in \mathcal{L}\left(L_{a}^{2}(\mathbb{D})\right), T \geq 0$. Then $T=\sum_{n=1}^{\infty} \sqrt{T e_{n}} \otimes\left(\sqrt{T e_{n}}\right)^{*}$, where $\left\{e_{n}\right\}$ is an orthonormal basis for $L_{a}^{2}(\mathbb{D})$, and the infinite sum means the limit of partial sums in the strong operator topology.

Proof. Define $P_{N}=\sum_{n=1}^{N} e_{n} \otimes e_{n}^{*}$. Clearly $P_{N} \uparrow I$ strongly. Thus $\sqrt{T} P_{N} \sqrt{T} \longrightarrow T$ strongly and $\sqrt{T} P_{N} \sqrt{T}=\sum_{n=1}^{N} \sqrt{T e_{n}} \otimes\left(\sqrt{T e_{n}}\right)^{*}$.

\section{THE OPERATOR $U_{a}$ AND CERTAin DistancE ESTIMATES}

In this section we propose some distance estimates for operators defined on the Bergman space. Such inequalities are useful in the theory of best approximation in $C^{*}$-algebras [5], complex interpolation [3], the theory of generalized inverses and operator approximation (see, [9], [10] and [1]). We obtain estimates for $\left\|U_{a}-T\right\|$ where $T \in \mathcal{L}\left(L_{a}^{2}(\mathbb{D})\right), T \geq 0$ and $a \in \mathbb{D}$.

It is well known [7] that if $T \in \mathcal{L}\left(L_{a}^{2}(\mathbb{D})\right)$ and there exists $\delta, 0<\delta<1$ such that $\left\|I-T^{n}\right\| \leq \delta<1$ for all $n \in \mathbb{N}$, then $T=I$, the identity operator. Thus it follows from this that $\left\|I-U_{a}\right\| \geq 1$ since $U_{a} \neq I$ and $U_{a}^{2}=I$. Further, since $U_{a}$ is not positive, it follows from [7] that there exists no positive integer $N$ such that $\left\|I-\frac{1}{n+1} \sum_{j=k}^{k+n} U_{a}^{j}\right\| \leq 1$ for all $n \geq N$ and $k \in \mathbb{N} \cup\{0\}$. But the following holds:

Theorem 3.1. Let $A$ be a positive operator in $\mathcal{L}\left(L_{a}^{2}(\mathbb{D})\right)$. Suppose for some a $\in$ $\mathbb{D},\left\|U_{a}-A\right\|<1$. Then $A$ is invertible. Further, $\|I-A\| \leq\left\|U_{a}-A\right\| \leq\|I+A\|$.

Proof. If $f \in L_{a}^{2}(\mathbb{D})$ and $\|f\|=1$ then

$$
\begin{aligned}
\left\|\left(U_{a}-A\right) f\right\|^{2} & =\left\langle\left(U_{a}-A\right) f,\left(U_{a}-A\right) f\right\rangle \\
& =\left\|U_{a} f\right\|^{2}+\|A f\|^{2}-\left\langle U_{a} f, A f\right\rangle-\left\langle A f, U_{a} f\right\rangle \\
& =\|f\|^{2}+\|A f\|^{2}-\left\langle f, U_{a}^{*} A f\right\rangle-\left\langle U_{a}^{*} A f, f\right\rangle \\
& =1+\|A f\|^{2}-\left\langle f, U_{a} A f\right\rangle-\left\langle U_{a} A f, f\right\rangle \\
& =1+\|A f\|^{2}-\overline{\left\langle U_{a} A f, f\right\rangle}-\left\langle U_{a} A f, f\right\rangle \\
& =1+\|A f\|^{2}-2 \operatorname{Re}\left\langle U_{a} A f, f\right\rangle \\
& \geq 1+\|A f\|^{2}-2\left|\left\langle U_{a} A f, f\right\rangle\right| \\
& \geq 1+\|A f\|^{2}-2\left\|U_{a} A f\right\|\|f\| \\
& =1+\|A f\|^{2}-2\|A f\|\|f\| \\
& =1+\|A f\|^{2}-2\|A f\|=(1-\|A f\|)^{2} .
\end{aligned}
$$


Now since $A$ is positive, $\inf _{\|f\|=1}\langle A f, f\rangle=\inf _{\|f\|=1}\|A f\|$ and $\sup _{\|f\|=1}\langle A f, f\rangle=\sup _{\|f\|=1}\|A f\|$ and we have

$$
\begin{aligned}
\left\|U_{a}-A\right\| & =\sup _{\|f\|=1}\left\|\left(U_{a}-A\right) f\right\| \\
& \geq \sup _{\|f\|=1}|1-\|A f\|| \\
& =\sup _{\|f\|=1}|1-\langle A f, f\rangle| \\
& =\sup _{\|f\|=1}|\langle(I-A) f, f\rangle|=\|I-A\| .
\end{aligned}
$$

Thus if $\left\|U_{a}-A\right\|<1$ then $\|I-A\|<1$ and this implies $A$ is invertible [4]. Further,

$$
\begin{aligned}
\left\|U_{a}-A\right\| & =\sup _{\|f\|=1}\left\|U_{a} f-A f\right\| \\
& \leq \sup _{\|f\|=1}(1+\|A f\|) \\
& =\sup _{\|f\|=1}\langle(I+A) f, f\rangle=\|I+A\| .
\end{aligned}
$$

Theorem 3.2. Let $T \in \mathcal{L}\left(L_{a}^{2}(\mathbb{D})\right)$. The following hold:

(i): If for some $a \in \mathbb{D},\left\|U_{a}-T\right\|<1$, then $T$ is invertible.

(ii): If $T$ is invertible and $\left\|U_{a}-r T\right\|=1$ for some real number $r>1$ and $a \in \mathbb{D}$, then $\left\|U_{a}-T\right\|<1$.

Proof. (i) The operator $U_{a}$ is a unitary operator and $U_{a}^{2}=I, U_{a}^{*}=U_{a}$. Since

$$
\left\|I-U_{a} T\right\|=\left\|U_{a}-T\right\|<1,
$$

the operator $U_{a} T$ is invertible. Hence $T$ is invertible as $T=U_{a}\left(U_{a} T\right)$. This completes the proof of (i). To prove (ii), we first show that if $S, R \in \mathcal{L}\left(L_{a}^{2}(\mathbb{D})\right)$ and $\|S-R\|=\|S\|+\|R\|$ then there exists a sequence $\left\{f_{n}\right\}$ of unit vectors in $\mathcal{L}\left(L_{a}^{2}(\mathbb{D})\right)$ such that $\lim _{n \rightarrow \infty}\left\|(\|R\| S+\|S\| R) f_{n}\right\|=0$. The converse holds if one of $S$ and $R$ is an isometric operator. To prove this we use the well known fact that if $f$ and $g$ are two vectors in a normed linear space such that $\|f+g\|=\|f\|+\|g\|$ then $\|a f+b g\|=a\|f\|+b\|g\|$ for any nonnegative real numbers $a$ and $b$. Suppose $S \neq 0, \quad R \neq 0,\|S-R\|=\|S\|+\|R\|$. Let $K=\frac{S}{\|S\|}$ and $L=\frac{-R}{\|R\|}$. Since $\|S-R\|=\|S\|+\|R\|$ we have $\|K+L\|=2$. So there exists a sequence $\left\{f_{n}\right\}$ of unit vectors such that $\lim _{n \rightarrow \infty}\left\|K f_{n}+L f_{n}\right\|=2$. This implies that $\lim _{n \rightarrow \infty}\left\|K f_{n}-L f_{n}\right\|=0$ as $\left\|K f_{n}\right\| \leq 1,\left\|L f_{n}\right\| \leq 1$ for all $n$ and by Parallelogram law

$$
\left\|K f_{n}+L f_{n}\right\|^{2}+\left\|K f_{n}-L f_{n}\right\|^{2}=2\left\|K f_{n}\right\|^{2}+2\left\|L f_{n}\right\|^{2} .
$$

It thus follows that $\lim _{n \rightarrow \infty}\left\|(\|R\| S+\|S\| R) f_{n}\right\|=0$. Conversely, let $S$ be an isometric operator and $\lim _{n \rightarrow \infty}\left\|(\|R\| S+R) f_{n}\right\|=0$ for some sequence $\left\{f_{n}\right\} \in L_{a}^{2}(\mathbb{D})$ 
of unit vectors. Then we have

$2\|R\| \geq\left\|(\|R\| S-R) f_{n}\right\| \geq 2\|R\|\left\|S f_{n}\right\|-\left\|(\|R\| S+R) f_{n}\right\| \rightarrow 2\|R\|$ as $n \rightarrow \infty$.

Hence, $\|(\|R\| S-R)\|=2\|R\|$, equivalently, $\|S-R\|=1+\|R\|$. Thus we obtain that if $S, R \in \mathcal{L}\left(L_{a}^{2}(\mathbb{D})\right)$ and $S$ is an isometric operator then $\|S-R\|=1+\|R\|$ if and only if there exists a sequence $\left\{f_{n}\right\}$ of unit vectors in $L_{a}^{2}(\mathbb{D})$ such that $\lim _{n \rightarrow \infty}\left\|(\|R\| S+R) f_{n}\right\|=0$. Notice that

$$
\begin{aligned}
\left\|(\|T\|(r-1) S+\|(r-1) S\| T) f_{n}\right\| & =\left\|(\|T\|(r-1) S+(r-1)\|S\| T) f_{n}\right\| \\
& =(r-1)\left\|(\|T\| S+T) f_{n}\right\| .
\end{aligned}
$$

It thus follows that $\|(r-1) S-T\|<(r-1)+\|T\|$ if and only if there exists no sequence $\left\{f_{n}\right\} \in L_{a}^{2}(\mathbb{D})$ of unit vectors such that $\lim _{n \rightarrow \infty}\left\|(\|T\| S+T) f_{n}\right\|=0$. Now we shall establish (ii). Suppose $T$ is invertible and $\left\|U_{a}-r T\right\|=1$ for some real number $r>1$. From the above argument it follows that there exists no sequence $\left\{f_{n}\right\} \in L_{a}^{2}(\mathbb{D})$ of unit vectors such that

$$
\lim _{n \rightarrow \infty}\left\|\left(\left\|U_{a}-r T\right\| U_{a}+\left(r T-U_{a}\right)\right) f_{n}\right\|=0
$$

if and only if

$$
\left\|(r-1) U_{a}-\left(r T-U_{a}\right)\right\|<(r-1)+\left\|r T-U_{a}\right\|=r .
$$

We thus show that if there exists no sequence $\left\{f_{n}\right\} \in L_{a}^{2}(\mathbb{D})$ of unit vectors such that $\lim _{n \rightarrow \infty}\left\|T f_{n}\right\|=0$ then

$$
r\left\|U_{a}-T\right\|=\left\|r U_{a}-r T\right\|=\left\|(r-1) U_{a}-\left(r T-U_{a}\right)\right\|<r .
$$

That is, $\left\|U_{a}-T\right\|<1$. Hence if $T$ is invertible and $\left\|U_{a}-r T\right\|=1$ for some $a \in \mathbb{D}$ and some real number $r>1$ then $\left\|U_{a}-T\right\|<1$.

Corollary 3.3. Let $T \in \mathcal{L}\left(L_{a}^{2}(\mathbb{D})\right)$. Then $\left\|U_{a}-T\right\|=1+\|T\|$ for some $a \in \mathbb{D}$ if and only if the operator $\|T\| U_{a}+T$ is not invertible.

Proof. Suppose $r>1=\|T\|$ and $V_{1}=\frac{U_{a}+T}{r}$. Then $T=r V_{1}-U_{a}$ and $\left\|U_{a}-r V_{1}\right\|=$ $\|T\|=1$. Thus $V_{1}=\frac{U_{a}+T}{r}$ is not invertible if and only if $\left\|U_{a}-\frac{U_{a}+T}{r}\right\| \geq 1$. But

$$
\left\|(r-1) U_{a}-T\right\| \leq(r-1)\left\|U_{a}\right\|+\|T\|=r .
$$

Thus $V_{1}$ is not invertible if and only if $\left\|(r-1) U_{a}-T\right\|=r$. That is, if and only if, $\left\|U_{a}-T\right\|=1+\|T\|$. That is, $r V_{1}=U_{a}+T$ is not invertible if and only if $\left\|U_{a}-T\right\|=1+\|T\|$. For the general case, suppose $T \in \mathcal{L}\left(L_{a}^{2}(\mathbb{D})\right)$. Then $\left\|\frac{T}{\|T\|}\right\|=1$. From the first part we obtain $U_{a}+\frac{T}{\|T\|}$ is not invertible if and only if

$$
\left\|U_{a}-\frac{T}{\|T\|}\right\|=1+\left\|\frac{T}{\|T\|}\right\|=2 .
$$

Thus $\|T\| U_{a}+T$ is not invertible if and only if \|\|$T\left\|U_{a}-T\right\|=2\|T\|$. That is, if and only if $\left\|U_{a}-T\right\|=1+\|T\|$. This completes the proof.

Corollary 3.4. Let $T \in \mathcal{L}\left(L_{a}^{2}(\mathbb{D})\right)$. Let $R=U_{a} T-T U_{a}$ for some $a \in \mathbb{D}$. Then $\|I-R\| \geq 1$. 
Proof. Notice that $\left\|U_{a}\right\|=1$ and the spectral radius of $U_{a}=r\left(U_{a}\right)=1$. In fact the spectrum of $U_{a}=\sigma\left(U_{a}\right)=\{-1,1\}$. Hence there is a sequence of unit vectors $f_{n} \in L_{a}^{2}(\mathbb{D}),(n=1,2,3, \cdots)$ such that $\left(U_{a}-I\right) f_{n} \rightarrow 0$. Now

$$
\|I-R\| \geq\left|\left\langle(I-R) f_{n}, f_{n}\right\rangle\right|=\left|1-\left\langle R f_{n}, f_{n}\right\rangle\right| \geq 1-\left|\left\langle R f_{n}, f_{n}\right\rangle\right| .
$$

The result follows if we can show that $\left\langle R f_{n}, f_{n}\right\rangle \rightarrow 0$. But

$$
\begin{aligned}
\left\langle R f_{n}, f_{n}\right\rangle & =\left\langle\left(\left(U_{a}-I\right) T-T\left(U_{a}-I\right)\right) f_{n}, f_{n}\right\rangle \\
& =\left\langle T f_{n},\left(U_{a}-I\right) f_{n}\right\rangle-\left\langle\left(U_{a}-I\right) f_{n}, T^{*} f_{n}\right\rangle .
\end{aligned}
$$

So

$$
\left|\left\langle R f_{n}, f_{n}\right\rangle\right| \leq\|T\|\left(\left\|\left(U_{a}-I\right) f_{n}\right\|+\left\|\left(U_{a}-I\right) f_{n}\right\|\right) \rightarrow 0 \text { as } n \rightarrow \infty
$$

The Schatten-Von Neumann class $S_{p}=S_{p}(H), 0<p<\infty$, consists of all operators $T \in \mathcal{L C}(H)$ such that

$$
\|T\|_{S_{p}}=\left(\sum_{n=0}^{\infty}\left(s_{n}(T)\right)^{p}\right)^{\frac{1}{p}}<\infty .
$$

Corollary 3.5. If $A$ is positive and $A \in \mathcal{L}\left(L_{a}^{2}(\mathbb{D})\right)$ and $U_{a}-A \in S_{p}, 0<p<\infty$ for some $a \in \mathbb{D}$, then $I-A \in S_{p}$.

Proof. Suppose $U_{a}-A \in S_{p}$ for some $a \in \mathbb{D}$. Then

$$
-A U_{a}+U_{a} A=\left(U_{a}-A\right) U_{a}-U_{a}\left(U_{a}-A\right) \in S_{p} .
$$

Hence

$$
I-A^{2}=\left(U_{a}-A\right)\left(U_{a}+A\right)+\left(A U_{a}-U_{a} A\right) \in S_{p}
$$

Since $A$ is positive, hence $I+A$ is invertible and so $I-A=\left(I-A^{2}\right)(I+A)^{-1} \in$ $S_{p}$

\section{TOEplitz Operators With BOUNDED HARMONIC SYMBOLS}

For $T \in \mathcal{L}\left(L_{a}^{2}(\mathbb{D})\right), T \geq 0$, let $W(T)=\{\langle T f, f\rangle:\|f\|=1\}$, the numerical range of $T$ and $w(T)=\sup \{|\langle T f, f\rangle|:\|f\|=1\}$, the numerical radius of $T$. It is well known that $W(T)$ is convex and its closure contains $\sigma(T)$. There are certain standard norm estimates like (see [1]), $w(T) \leq\|T\| \leq 2 w(T)$ and $w\left(T^{n}\right) \leq$ $w(T)^{n}, n \in \mathbb{N}$. It is not hard to check that for $T \in \mathcal{L}\left(L_{a}^{2}(\mathbb{D})\right)$, the range of $\overline{\widetilde{T}}$ is contained in $W(T)$ and $\|\widetilde{T}\|_{\infty} \leq w(T)$. Thus if $\phi \in L^{\infty}(\mathbb{D})$, then $\|\widetilde{\phi}\|_{\infty}=$ $\left\|\widetilde{T_{\phi}}\right\|_{\infty} \leq w\left(T_{\phi}\right) \leq\left\|T_{\phi}\right\| \leq\|\phi\|_{\infty}$. The natural question that arises at this point is the following: Is there a real number $M>0$ with $\left\|T_{\phi}\right\| \leq M\|\widetilde{\phi}\|_{\infty}$ for all $\phi \in L^{\infty}(\mathbb{D})$ and if so what are the distance estimates for $\left\|T_{\phi}-T_{\psi}\right\|$ and $\left\|U_{a}-T_{\phi}\right\|$ where, $\phi, \psi \in L^{\infty}(\mathbb{D})$ ? In this section, we are concerned only with Toeplitz operators with bounded harmonic symbols and obtained such distance estimates.

Theorem 4.1. If $\phi, \psi \in h^{\infty}(\mathbb{D})$ and $T_{\phi}, T_{\psi} \geq 0$, then

(i): For all $a \in \mathbb{D},\left\|T_{\psi \circ \phi_{a}}-T_{\phi}\right\| \leq \max \left\{\|\phi\|_{\infty},\|\psi\|_{\infty}\right\}$.

(ii): $\left\|T_{\phi} T_{\psi}-T_{\psi} T_{\phi}\right\| \leq\left(\max \left\{\|\phi\|_{\infty},\|\psi\|_{\infty}\right\}\right)^{2}$. 
(iii): If $r>0$ and $T_{\phi+\psi} \geq r I \geq 0$, then $\left\|T_{\phi} U_{a}+U_{a} T_{\psi}\right\| \geq r$.

Proof. (i) Let $\widetilde{\phi}(z)=\left\langle T_{\phi} k_{z}, k_{z}\right\rangle$, where $k_{z}$ is the normalized reproducing kernel for the Hilbert space $L_{a}^{2}(\mathbb{D})$. Hence $\widetilde{\phi}(z)=\int_{\mathbb{D}} \phi(w)\left|k_{z}(w)\right|^{2} d A(w), z \in \mathbb{D}$. Since $\phi_{z}^{\prime}=-k_{z}$, making a change of variable we also have $\widetilde{\phi}(z)=\int_{\mathbb{D}} \phi\left(\phi_{z}(w)\right) d A(w)=$ $\phi\left(\phi_{z}(0)\right)=\phi(z), z \in \mathbb{D}$. Thus $|\phi(z)|=|\widetilde{\phi}(z)|=\left|\left\langle T_{\phi} k_{z}, k_{z}\right\rangle\right| \leq\left\|T_{\phi}\right\|$ for all $z \in \mathbb{D}$. Hence $\|\phi\|_{\infty} \leq\left\|T_{\phi}\right\|$. Now since the Bergman projection has norm 1, we have $\left\|T_{\phi}\right\|=\|\phi\|_{\infty}$.

Suppose $a \in \mathbb{D}$. Now on $L_{a}^{2}(\mathbb{D}) \oplus L_{a}^{2}(\mathbb{D})$, let

$$
M=\left(\begin{array}{cc}
T_{\phi} & 0 \\
0 & T_{\psi}
\end{array}\right) \text { and } N=\left(\begin{array}{cc}
0 & U_{a} \\
0 & 0
\end{array}\right) .
$$

Then $M \geq 0$ and $M N-N M=\left(\begin{array}{cc}0 & T_{\phi} U_{a}-U_{a} T_{\psi} \\ 0 & 0\end{array}\right)$. From [2] we have

$$
\begin{aligned}
\left\|T_{\psi \circ \phi_{a}}-T_{\phi}\right\| & =\left\|T_{\phi} U_{a}-U_{a} T_{\psi}\right\|=\|M N-N M\| \leq\|M\|\|N\| \\
& =\max \left\{\left\|T_{\phi}\right\|,\left\|T_{\psi}\right\|\right\}\left\|U_{a}\right\|=\max \left\{\|\phi\|_{\infty},\|\psi\|_{\infty}\right\} .
\end{aligned}
$$

This completes the proof of (i). To prove (ii), observe that

$$
\begin{aligned}
2\left(T_{\psi} T_{\phi}-T_{\phi} T_{\psi}\right) & =\left(T_{\phi}+T_{\psi}\right)\left(T_{\phi}-T_{\psi}\right)-\left(T_{\phi}-T_{\psi}\right)\left(T_{\phi}+T_{\psi}\right) \\
& =T_{\phi+\psi} T_{\phi-\psi}-T_{\phi-\psi} T_{\phi+\psi} .
\end{aligned}
$$

Since $T_{\phi+\psi}$ is positive and $\left\|T_{\phi+\psi}\right\|=\|\phi+\psi\|_{\infty}$, we have from [2] that

$$
\begin{aligned}
2\left\|T_{\psi} T_{\phi}-T_{\phi} T_{\psi}\right\| & \leq\left\|T_{\phi+\psi}\right\|\left\|T_{\phi-\psi}\right\|=\|\phi+\psi\|_{\infty}\left\|T_{\phi}-T_{\psi}\right\| \\
& =\|\phi+\psi\|_{\infty} \max \left\{\|\phi\|_{\infty},\|\psi\|_{\infty}\right\} .
\end{aligned}
$$

Thus

$\left\|T_{\psi} T_{\phi}-T_{\phi} T_{\psi}\right\| \leq \frac{1}{2}\left\{\|\phi\|_{\infty}+\|\psi\|_{\infty}\right\} \max \left\{\|\phi\|_{\infty},\|\psi\|_{\infty}\right\} \leq\left(\max \left\{\|\phi\|_{\infty},\|\psi\|_{\infty}\right\}\right)^{2}$.

This completes the proof of (ii). To prove (iii), notice that the spectral radius of $U_{a}=r\left(U_{a}\right)=1$ and $\left\|U_{a}\right\|=1$ and $\sigma\left(U_{a}\right)=\{-1,1\}$. Hence there is a sequence of unit vectors $\left\{f_{n}\right\} \in L_{a}^{2}(\mathbb{D})$ such that $\left(U_{a}-I\right) f_{n} \rightarrow 0$. Now

$$
\begin{aligned}
\left\|T_{\phi} U_{a}+U_{a} T_{\psi}\right\| & \geq\left|\left\langle\left(T_{\phi} U_{a}+U_{a} T_{\psi}\right) f_{n}, f_{n}\right\rangle\right| \\
& =\left|\left\langle T_{\phi}\left(U_{a}-I\right) f_{n}, f_{n}\right\rangle+\left\langle\left(U_{a}-I\right) T_{\psi} f_{n}, f_{n}\right\rangle+\left\langle\left(T_{\phi}+T_{\psi}\right) f_{n}, f_{n}\right\rangle\right| \\
& =\left|\left\langle\left(U_{a}-I\right) f_{n}, T_{\phi} f_{n}\right\rangle+\left\langle T_{\psi} f_{n},\left(U_{a}-I\right) f_{n}\right\rangle+\left\langle\left(T_{\phi}+T_{\psi}\right) f_{n}, f_{n}\right\rangle\right| \\
& \geq\left|\left\langle\left(T_{\phi}+T_{\psi}\right) f_{n}, f_{n}\right\rangle\right|-\left[\left\|\left(U_{a}-I\right) f_{n}\right\|\left\|T_{\phi} f_{n}\right\|+\left\|T_{\psi} f_{n}\right\|\left\|\left(U_{a}-I\right) f_{n}\right\|\right] \\
& \geq r-r_{n}
\end{aligned}
$$

where $r_{n}$ is a term depending on $n$ and $r_{n} \rightarrow 0$ as $n \rightarrow \infty$. Hence

$$
\left\|T_{\phi} U_{a}+U_{a} T_{\psi}\right\| \geq r .
$$


Theorem 4.2. (i): If $\phi \in L^{\infty}(\mathbb{D})$ and $T_{\phi}^{2}+T_{\phi \circ \phi_{a}}^{2}=2 T_{\phi \circ \phi_{a}} T_{\phi}$ for some $a \in \mathbb{D}$, then $\lim _{n \rightarrow \infty} \frac{\left\|T_{\phi}^{n-1}\left(T_{\phi \circ \phi_{a}-\phi}\right)\right\|}{\left\|T_{\phi}^{n-1}\right\|}=0, T_{\phi \circ \phi_{a}-\phi}$ is not invertible and $\| U_{a}-$ $T_{\phi \circ \phi_{a}-\phi} \| \geq 1$.

(ii): If $\phi \in L^{\infty}(\mathbb{D})$ and $T_{\phi}$ is positive, then also $\left\|U_{a}-T_{\phi \circ \phi_{a}-\phi}\right\| \geq 1$.

Proof. (i) Suppose $T_{\phi}^{2}+T_{\phi \circ \phi_{a}}^{2}=2 T_{\phi \circ \phi_{a}} T_{\phi}$ for some $a \in \mathbb{D}$. Then

$$
T_{\phi \circ \phi_{a}} T_{\phi}-T_{\phi}^{2}=T_{\phi \circ \phi_{a}}^{2}-T_{\phi \circ \phi_{a}} T_{\phi}
$$

That is,

$$
\begin{aligned}
U_{a} T_{\phi} U_{a} T_{\phi}-T_{\phi}^{2} & =\left(U_{a} T_{\phi} U_{a}\right)\left(U_{a} T_{\phi} U_{a}\right)-\left(U_{a} T_{\phi} U_{a}\right) T_{\phi} \\
& =U_{a} T_{\phi}^{2} U_{a}-\left(U_{a} T_{\phi} U_{a}\right) T_{\phi} .
\end{aligned}
$$

Hence $T_{\phi} U_{a} T_{\phi}-U_{a} T_{\phi}^{2}=T_{\phi}^{2} U_{a}-T_{\phi} U_{a} T_{\phi}$. That is, $\left(T_{\phi} U_{a}-U_{a} T_{\phi}\right) T_{\phi}=T_{\phi}\left(T_{\phi} U_{a}-\right.$ $\left.U_{a} T_{\phi}\right)$. Thus $T_{\phi}$ commutes with $T_{\phi} U_{a}-U_{a} T_{\phi}$. Suppose there exists a $m \in \mathbb{Z}_{+}$such that $T_{\phi}^{m}=0$. Now since

$$
\begin{aligned}
T_{\phi}^{n} U_{a}-U_{a} T_{\phi}^{n} & =\sum_{i=0}^{n-1} T_{\phi}^{n-1-i}\left(T_{\phi} U_{a}-U_{a} T_{\phi}\right) T_{\phi}^{i} \\
& =n T_{\phi}^{n-1}-\sum_{i=0}^{n-1} T_{\phi}^{n-1-i}\left(I-\left(T_{\phi} U_{a}-U_{a} T_{\phi}\right)\right) T_{\phi}^{i},
\end{aligned}
$$

we have $0=T_{\phi}^{m} U_{a}-U_{a} T_{\phi}^{m}=m T_{\phi}^{m-1}\left(T_{\phi} U_{a}-U_{a} T_{\phi}\right)$ and hence

$$
\lim _{n \rightarrow \infty} \frac{\left\|T_{\phi}^{n-1}\left(T_{\phi} U_{a}-U_{a} T_{\phi}\right)\right\|}{\left\|T_{\phi}^{n-1}\right\|}=\lim _{n \rightarrow \infty} \frac{\left\|T_{\phi}^{n-1}\left(T_{\phi \circ \phi_{a}-\phi}\right)\right\|}{\left\|T_{\phi}^{n-1}\right\|}=0 .
$$

If there does not exist $m \in \mathbb{Z}_{+}$such that $T_{\phi}^{m}=0$ then the inequality

$$
\begin{aligned}
n\left\|T_{\phi}^{n-1} T_{\phi \circ \phi_{a}-\phi}\right\| & =n\left\|T_{\phi}^{n-1}\left(T_{\phi} U_{a}-U_{a} T_{\phi}\right)\right\|=\left\|T_{\phi}^{n} U_{a}-U_{a} T_{\phi}^{n}\right\| \\
& \leq 2\left\|T_{\phi}\right\|\left\|U_{a}\right\|\left\|T_{\phi}^{n-1}\right\|=2\left\|T_{\phi}\right\|\left\|T_{\phi}^{n-1}\right\|
\end{aligned}
$$

implies that $\frac{\left\|T_{\phi}^{n-1}\left(T_{\phi \circ \phi_{a}-\phi}\right)\right\|}{\left\|T_{\phi}^{n-1}\right\|} \rightarrow 0$ as $n \rightarrow \infty$. Suppose now that $T_{\phi \circ \phi_{a}-\phi}$ is invertible and $\left\|T_{\phi \circ \phi_{a}-\phi}^{-1}\right\| \leq C$ for some constant $C>0$. Then

$$
\begin{aligned}
1 & =\frac{\left\|T_{\phi}^{n-1} T_{\phi \circ \phi_{a}-\phi} T_{\phi \circ \phi_{a}-\phi}^{-1}\right\|}{\left\|T_{\phi}^{n-1}\right\|} \\
& \leq \frac{\left\|T_{\phi}^{n-1} T_{\phi \circ \phi_{a}-\phi}\right\|\left\|T_{\phi \circ \phi_{a}-\phi}^{-1}\right\|}{\left\|T_{\phi}^{n-1}\right\|} \\
& \leq \frac{C\left\|T_{\phi}^{n-1} T_{\phi \circ \phi_{a}-\phi}\right\|}{\left\|T_{\phi}^{n-1}\right\|} \leq \frac{2 C\left\|T_{\phi}\right\|}{n} .
\end{aligned}
$$

Taking limit as $n \rightarrow \infty$ both the sides we obtain a contradiction. Hence $T_{\phi \circ \phi_{a}-\phi}$ is not invertible. That is, $U_{a} T_{\phi} U_{a}-T_{\phi}=U_{a}\left(T_{\phi} U_{a}-U_{a} T_{\phi}\right)$ is not invertible. 
Hence $T_{\phi} U_{a}-U_{a} T_{\phi}$ is not invertible and $\left\|I-\left(T_{\phi} U_{a}-U_{a} T_{\phi}\right)\right\| \geq 1$. That is,

$$
\left\|U_{a}-T_{\phi \circ \phi_{a}-\phi}\right\|=\left\|U_{a}-T_{\phi \circ \phi_{a}}+T_{\phi}\right\|=\left\|I-\left(T_{\phi} U_{a}-U_{a} T_{\phi}\right)\right\| \geq 1 .
$$

This completes the proof of (i). To prove (ii), assume $T_{\phi}$ is positive. Then $\left\|T_{\phi}^{m}\right\|=\left\|T_{\phi}\right\|^{m}$ for all $m \in \mathbb{Z}_{+}$. Thus it follows that

$$
\begin{aligned}
n\left\|T_{\phi}\right\|^{n-1} & =n\left\|T_{\phi}^{n-1}\right\| \\
& \leq\left\|T_{\phi}^{n} U_{a}-U_{a} T_{\phi}^{n}\right\|+\left\|\sum_{i=0}^{n-1} T_{\phi}^{n-1-i}\left(I-\left(T_{\phi} U_{a}-U_{a} T_{\phi}\right)\right) T_{\phi}^{i}\right\| \\
& \leq 2\left\|T_{\phi}\right\|\left\|U_{a}\right\|\left\|T_{\phi}^{n-1}\right\|+\left\|I-\left(T_{\phi} U_{a}-U_{a} T_{\phi}\right)\right\| \sum_{i=0}^{n-1}\left\|T_{\phi}^{n-1-i}\right\|\left\|T_{\phi}^{i}\right\| \\
& =2\left\|T_{\phi}\right\|\left\|T_{\phi}\right\|^{n-1}+\left\|I-\left(T_{\phi} U_{a}-U_{a} T_{\phi}\right)\right\| \sum_{i=0}^{n-1}\left\|T_{\phi}\right\|^{n-1-i}\left\|T_{\phi}\right\|^{i} \\
& =2\left\|T_{\phi}\right\|^{n}+n\left\|I-\left(T_{\phi} U_{a}-U_{a} T_{\phi}\right)\right\|\left\|T_{\phi}\right\|^{n-1} .
\end{aligned}
$$

Thus

$$
1 \leq \frac{2}{n}\left\|T_{\phi}\right\|+\left\|I-\left(T_{\phi} U_{a}-U_{a} T_{\phi}\right)\right\| \text { for all } n \in \mathbb{Z}_{+} .
$$

If now $\left\|I-\left(T_{\phi} U_{a}-U_{a} T_{\phi}\right)\right\|<1$ then we get a contradiction. Hence

$$
\left\|U_{a}-T_{\phi \circ \phi_{a}-\phi}\right\|=\left\|I-\left(T_{\phi} U_{a}-U_{a} T_{\phi}\right)\right\| \geq 1
$$

\section{REFERENCES}

1. A.W. Marshall and I. Olkin, Inequalities: theory of majorization and its application, Academic Press, New York, 1979.

2. F. Kittaneh, Norm inequalities for commutators of positive operators and applications, Math. Z. 258 (2008), 845-849.

3. G. Pisier, Similarity problems and completely bounded maps, 2nd edition, Lecture Notes in Math. 1618, Springer-Verlag, Berlin, 2001.

4. I. Gohberg and S. Goldberg, Basic operator theory, Birkhauser, 1981.

5. K. Davidson and S.C. Power, Best approximation in $C^{*}$-algebras, J. Reine Angew. Math. 368 (1986), 43-62.

6. K. Zhu, On certain unitary operators and composition operators, Proc. Symp. Pure Math. 51 (1990), 371-385.

7. M. Nagisa and S. Wada, Averages of operators and their positivity, Proc. Amer. Math. Soc. 126 (1998), no. 2, 499-506.

8. N. Das, The Berezin transform of bounded linear operators, J. Indian Math. Soc. 76 (2009), 47-60.

9. P.J. Maher, Some operator inequalities concerning generalized inverses, Illinois J. Math. 34 (1990), 503-514.

10. P.J. Maher, Some norm inequalities concerning generalized inverses, Linear Algebra Appl. 174 (1992), 99-110.

11. S. Axler and D. Zheng, The Berezin transform on the Toeplitz algebra, Studia Math. 127 (1998), no. 2, 113-136. 
${ }^{1}$ P.G.Department of Mathematics, Utkal University, Vani Vihar, Bhubaneswar751004, Odisha, INDIA.

E-mail address: namitadas440@yahoo.co.in

${ }^{2}$ School of Applied Sciences (Mathematics), Kitt University, Campus-3(Kathajori Campus), Bhubaneswar-751024, Odisha, India.

E-mail address: smita_782006@yahoo.co.in 